\title{
Space-Time Autocoding: Arbitrarily Reliable Communication in a Single Fading Interval
}

\author{
Thomas L. Marzetta, Bertrand Hochwald and Babak Hassibi \\ Mathematical Sciences Center \\ Lucent Technologies \\ 600 Mountain Avenue \\ Murray Hill, NJ 07974 \\ e-mail: $\{t l m, h o c h w a l d, h a s s i b i\}$ @research bell-labs.com
}

\begin{abstract}
Prior treatments of space-time communications in Rayleigh flat fading generally assume that channel coding covers either one fading interval-in which case there is a nonzero "outage capacity"or multiple fading intervals -in which case there is a nonzero Shannon capacity. However, we establish conditions under which channel codes span only one fading interval and yet are arbitrarily reliable. In short, space-time signals are their own channel codes. We call this phenomenon space-time autocoding, and the accompanying capacity the space-time autocapacity.
\end{abstract}

Let an $M$-transmitter-antenna, $N$-receiver-antenna Rayleigh flat fading channel be characterized by an $M \times N$ matrix of independent propagation coefficients, distributed as zero-mean, unit-variance complex Gaussian random variables. This propagation matrix is unknown to the transmitter, remains constant during a $T$-symbol coherence interval, and there is a fixed total transmit power. Let the coherence interval and number of transmitter antennas be related as $T=\beta M$ for some $\beta$. A $T \times M$ matrix-valued signal, associated with $R \cdot T$ bits of information for some rate $R$ is transmitted during the $T$-symbol coherence interval. Then there is a positive space-time autocapacity $C_{a}$ such that for all $R<C_{a}$, the block probability of error goes to zero as the pair $(T, M) \rightarrow \infty$ such that $T / M=\beta$. The autocoding effect occurs whether or not the propagation matrix is known to the receiver, and $C_{a}=N \log (1+\rho)$ in either case independently of $\beta$, where $\rho$ is the expected SNR at each receiver antenna. Lower bounds on the cutoff rate derived from random Unitary Space-Time signals suggest that the autocoding effect manifests itself for relatively small values of $T$ and $M$. For example within a single coherence interval of duration $T=16$, for $M=7$ transmitter antennas and $N=4$ receiver antennas, and an $18 \mathrm{~dB}$ expected SNR, a total of 80 bits (corresponding to rate $R=5$ ) can theoretically be transmitted with a block probability of error less than $10^{-9}$, all without any training or knowledge of the propagation matrix.

A complete copy of this paper is available on the web at http://mars.bell-labs.com.

\section{REFERENCES}

[1] B. Hochwald, T. Marzetta and B. Hassibi, "Space-time autocoding," submitted to IEEE Trans. Info. Theory. Also Bell Labs. tech. report, Nov. 1999 